\title{
Expression Analysis of Sound Vibration-Regulated Genes by Touch Treatment in Arabidopsis
}

\author{
Ritesh Ghosh1, Mayank A. Gururani2, Lakshmi N. Ponpandian', Ratnesh C. Mishra1, \\ Soo-Chul Park ${ }^{3}$, Mi-Jeong Jeong ${ }^{3}$ and Hanhong Bae ${ }^{1 *}$
}

${ }^{1}$ Department of Biotechnology, Yeungnam University, Gyeongsan, South Korea, ${ }^{2}$ Department of Biology, College of Science, United Arab Emirates University, Al Ain, United Arab Emirates, ${ }^{3}$ National Institute of Agricultural Sciences, Rural

Development Administration, Wanju, South Korea

OPEN ACCESS

Edited by:

Vicenta Salvador Recatala, Ronin Institute, USA

Reviewed by:

Heidi M. Appel,

University of Missouri, USA

Elizabeth Haswell,

Washington University in Saint Louis,

USA

${ }^{*}$ Correspondence: Hanhong Bae

hanhongbae@ynu.ac.kr

Specialty section:

This article was submitted to

Plant Physiology,

a section of the journal

Frontiers in Plant Science

Received: 14 October 2016 Accepted: 18 January 2017

Published: 31 January 2017

Citation:

Ghosh R, Gururani MA, Ponpandian LN, Mishra RC,

Park S-C, Jeong M-J and Bae H (2017) Expression Analysis of Sound Vibration-Regulated Genes by Touch

Treatment in Arabidopsis.

Front. Plant Sci. 8:100.

doi: 10.3389/fpls.2017.00100
Sound vibration (SV) is considered to be a mechanical stimulus which gives rise to various physiological and molecular changes in plants. Previously, we identified 17 SVregulated genes (SRGs) which were up-regulated by SV treatments in Arabidopsis. Here, we analyzed the expression pattern of similar genes after an exposure of 500 Hertz at 80 decibels, for various time periods. Simultaneously, we confirmed the SV-mediated expression of these genes under lighted condition as many of them were reported to be dark-induced. For this, we designed an improved SV treatment chamber. Additionally, we checked the electrolyte leakage (EL), photosynthetic performance and expression of mechanosensitive (MS) ion channel genes after 5 days of SV treatment in the illuminated chamber. EL was higher, and the photosynthetic performance index was lower in the SV-treated plants compared to control. Seven out of the $13 \mathrm{MS}$ ion channel genes were differentially expressed after the SV treatment. Simultaneously, we checked the touchmediated expression pattern of 17 SRGs and 13 MS ion channel genes. The distinct expression pattern of 6 SRGs and 1 MS ion channel gene generate an idea that SV as a stimulus is different from touch. Developmental stage-specific expression profiling suggested that the majority of the SRGs were expressed spatiotemporally in different developmental stages of Arabidopsis, especially in imbibed seed, seedlings and leaves.

Keywords: sound vibration, touch, mechano-stimulus, MS ion channel, electrolyte leakage, photosynthesis

\section{INTRODUCTION}

Sound vibration (SV) is considered to be a mechanical stimulus which can create the thigmomorphogenetic response in plants (Telewski, 2006). Available evidences suggest that the interaction between SV and plants is relevant both in ecological as well as environmental context. For instance, the phenomenon of 'Buzz Pollination' has been noted in number of plant species which indicates the ecological relevance of SV. Buzz- pollinated plants release pollen from anthers only at a particular frequency produced by bee's buzz (De Luca and Vallejo-Marin, 2013). Similarly, plants' responsiveness to environmental sound has been shown recently. Pretreatment with vibrations caused by chewing sound of caterpillar has been noted to elicit plant defense against herbivore (Appel and Cocroft, 2014). This advocates the relevance of natural SV in plants' defense. In addition, several other plausible environmental significance of SV has been discussed by Mishra et al. (2016). Taken together, it is amply clear that like other physical factors SV is ecologically and/or environmentally significant to plants. 
Experimentally, previous studies have shown the various effects of synthetic single frequency SV on plants (Hassanien et al., 2014). SV has the ability to alter antioxidant activities, calcium flux, sugar and ATP contents, hormonal modulation and plasmalemma architecture in plants (Mishra et al., 2016). SV-induced antioxidant (like- catalase, superoxide dismutase, and ascorbate peroxidase) activity was noted in chrysanthemum seedlings and hazel cells (Xiujuan et al., 2003; Safari et al., 2013). Increased ATP content in the SV-treated Actinidia chinensis callus suggests that SV can alter the energy metabolism (Xiaocheng et al., 2003). SV-induced changes in levels of phytohormones (like- auxin, cytokinin, and salicylic acid) were previously reported in Arabidopsis plant, Chrysanthemum callus, and protocorm-like bodies of Dendrobium (Bochu et al., 2004; Wei et al., 2012; Ghosh et al., 2016). Besides that, beneficial effects of SV were noted in terms of disease resistance, crop yield, callus regeneration and plant growth (Hassanien et al., 2014). A growing body of recent evidence suggests the existence of sophisticated molecular mechanisms for SV perception and signal transduction in plants. Despite this, however, there exists a huge gap in our understanding regarding the SV-mediated molecular alterations in the cellular milieu, which is a prerequisite to gain insight into SV-mediated plant development. Necessitated by this, we had previously investigated the global transcriptomic and proteomic changes in Arabidopsis thaliana upon treatment with SV of five different frequencies (250, 500, 1000, 2000, $3000 \mathrm{~Hz}$ ) with constant amplitude to bridge this gap (Ghosh et al., 2016). Several genes were noted to be differentially expressed after SV treatment, and it was noted that $500 \mathrm{~Hz}$ for $1 \mathrm{~h}$ has maximum impact on cellular processes in Arabidopsis. Additionally, 17 genes were further confirmed by real-time PCR analysis, which are termed as SV-regulated genes (SRGs).

Another well-known mechanical stimulus which alters the physiology of plants at various levels is touch. Touch-mediated growth retardation, calcium spiking, reactive oxygen species generation, hormonal modulation and gene expression were previously reported (Braam, 2005; Chehab et al., 2011). It has been demonstrated that repetitive touch treatment can prime plants, which subsequently alter the plant defense against fungal pathogen and herbivore (Chehab et al., 2012). Previously, we hypothesized that SV and touch share some common mechanosensitive (MS) signaling events, as many of the touchregulated genes were induced by SV (Ghosh et al., 2016). Therefore, it was concluded that touch-mediated expression profiling of SRGs is necessary to strengthen this idea. In the present study, we investigated the expression of SRGs after the touch treatment. Simultaneously, we checked the expression pattern of similar genes after exposure of $500 \mathrm{~Hz}$ for various time periods, and investigated their transcript levels in various developmental stages. Additionally, we have designed an improved SV treatment chamber, and cross-confirmed the SV-mediated expression of SRGs in lighted conditions. Simultaneously, this chamber was used to investigate the effect of long-term SV treatment on physiological responses like electrolyte leakage (EL) and photosynthesis.

It was hypothesized that plant MS ion channels have important roles in mechanical stress perception and signaling
(Monshausen and Haswell, 2013; Mishra et al., 2016). In plants, broadly, three major groups of MS ion channels were reported: MS channel of small conductance-like (MSL), Mid1complementing activity family (MCA) and Piezo (Monshausen and Haswell, 2013). MSLs in Arabidopsis are homologous to Escherichia coli MS channel of small conductance (MscS) protein which provides the rapid release of osmolytes from cells in response to the increased membrane tension. MCA and Piezo belong to the putative stretch-activated $\mathrm{Ca}^{2+/-}$ permeable channels. Various electrophysiological, pharmacological and molecular-genetic analyses identified the importance of MSL9, MSL 10 and MCA1 for mechanosensitive activity in Arabidopsis (Nakagawa et al., 2007; Haswell et al., 2008). Additionally, the functions of MSLs in various biological processes, likechloroplast shaping, cell death signaling, and pollen germination, were previously reported (Haswell and Meyerowitz, 2006; Veley et al., 2014; Hamilton et al., 2015). Here, we investigated the expression level of MS ion channels after 5 days of continuous SV treatment. Simultaneously, we checked the transcript levels of these MS channel genes after repetitive touch treatments for 5 days.

In summary, we investigated the expression of 17 SRGs and 13 MS-ion channel genes after SV and touch treatments. Simultaneously, we have checked the developmental stagespecific expression pattern of 17 SRGs. In addition, EL and photosynthetic performance were analyzed to check the SVmediated physiological response after the long exposure.

\section{MATERIALS AND METHODS}

\section{Plant Materials and Growth Condition}

Arabidopsis thaliana (Col-0 ecotype) seeds were placed in pots containing artificial soil (Punong, Korea) and stratified for 2 days in the dark at $4^{\circ} \mathrm{C}$ for homogenous germination. Subsequently, pots were transferred to the growth room and seedlings were allowed to grow under continuous light $\left(\sim 150 \pm 10 \mu \mathrm{mol} \mathrm{m}^{-2}\right.$ $\mathrm{s}^{-1}$ ) at $22 \pm 1^{\circ} \mathrm{C}$. The growing seedlings received supplements with nutrients (Bio-nex, Korea) mixed in water at every 3-day interval.

\section{SV Treatment under Dark Conditions}

Twenty-day-old Arabidopsis plants were transferred to a soundproof chamber and subjected to $500 \mathrm{~Hz}$ at $80 \mathrm{~dB}$ (adjusted manually) for four different time periods (10, $30 \mathrm{~m}, 1$ and $2 \mathrm{~h}$ ) including the $1 \mathrm{~h}$ treatment as analyzed previously (Ghosh et al., 2016). A $1 \mathrm{~h}$ treatment was included for further confirming the stringency of our results. Control plants were kept in a similar chamber without SV treatment. The sound-proof chamber was customized by Korea Scientific Technique Industry (Korea) according to Jeong et al. (2008). This sound-proof chamber was not equipped with a light source and temperature controller. Sound intensity of the growth room, as recorded by a sound intensity meter TES-1350A (Pusung, Korea), was noted to be $75 \pm 2 \mathrm{~dB}$. The sound intensity within the chamber was recorded to be $40 \mathrm{~dB}$. The Adobe Audition version 3.0 software (USA) was used for generation of single frequency sound. After the 
SV treatment, rosette samples were harvested for quantitative real-time PCR (qRT-PCR) analysis.

\section{SV Treatment under Lighted Condition}

Thirteen-day-old plants were covered with a transparent plastic dome to protect them from mechanical perturbation caused by direct air flow within the plant growth chamber. Two sides of the dome were cut manually to make a big hole, and placed perpendicularly to the air flow channel. Additionally, eight small holes were made on the top of the dome for gentle aeration. Plants covered by the dome were placed on a thick sponge cushion within the chamber to reduce the effect of mechanical vibration of the compressor. This set-up was maintained for 2 days under continuous light $\left(\sim 150 \pm 10 \mu \mathrm{mol} \mathrm{m} \mathrm{m}^{-2} \mathrm{~s}^{-1}\right)$ at $22 \pm 1{ }^{\circ} \mathrm{C}$ within the growth chamber for the plants to get acclimatized. The plant growth chamber was equipped with a speaker (Sammi, Korea) and provision for watering from outside. A schematic diagram of this specialized plant growth chamber has been shown in Supplementary Figure S1. After the acclimatization, $1 \mathrm{~h} \mathrm{SV}$ treatment $(500 \mathrm{~Hz}, 100 \pm 1 \mathrm{~dB})$ was given under continuous illumination. The intensity of background noise within the chamber was $82 \pm 2 \mathrm{~dB}$. Control plants were acclimatized under similar condition without SV treatment. For long-term SV treatment, 15-day-old plants were transferred to this specialized plant growth chamber and treated continuously for 5 days. Plants were regularly watered upto 18 days. After 5 days, rosette leaves from 20-day-old SV-treated plants were harvested and used for the expression analysis of MS ion channel genes, EL and photosynthesis. Two independent experiments were performed by swapping the chambers between control and treatment (indicated as set 1 and 2 in the figures) to normalize the chamber effects and add more precision in our data. For a better representation of treatment method, a schematic diagram has been shown in Figure 1.

\section{Touch-Mediated Expression Profiling of SRGs and MS Ion Channel Genes}

Gentle touch treatment was given to the 18-day-old plants in the growth room, as mentioned previously (Lee et al., 2005); 3-4 mature rosette leaves per plant were gently bent back and forth (four times) manually, and the samples (all touched and untouched leaves in a rosette) were harvested at 5, 15, $30 \mathrm{~m}$ and $1 \mathrm{~h}$ after the touch treatments. The control sample was harvested before the touch treatments. For long-term treatments, the 15-day-old plants were repeatedly touched (twice per day at $10 \mathrm{~h}$ interval) for 5 days. After 5 days, rosette leaves (both touched and untouched) from 20-day-old touch-treated plants were harvested and used for the expression analysis of MS ion channel genes. At the same time, 20-day-old untouched plants were harvested as control sample. Two independent experiments with long-term touch treatments were performed and results for both have been shown (indicated as set 1 and 2 in the figures).

\section{Developmental Stage-Specific Samples Collection}

Rosette leaves (two types, young and mature), cauline leaves, young flowers (green and unopened buds) and mature flowers (white and fully opened), were harvested from 23-day-old plants. Samples from four different developmental stages (root, stem, young green pods and matured green pods) were harvested from 35-day-old plants. Ripening pods (fully developed and yellowish green in color) were collected from 38-day-old plants. Fifty to 100 seeds were washed twice, followed by soaking in water, and kept at $4^{\circ} \mathrm{C}$ for 2 days in dark. Imbibed seeds were subsequently transferred to the growth room under light for $10 \mathrm{~h}$ before harvesting. For the seedling sample, seeds were surface-sterilized and placed on plates containing $1 \times$ Murashige and Skoog (MS) salts and $0.4 \%$ phytagel (Sigma, USA), pH5.8. Subsequently,

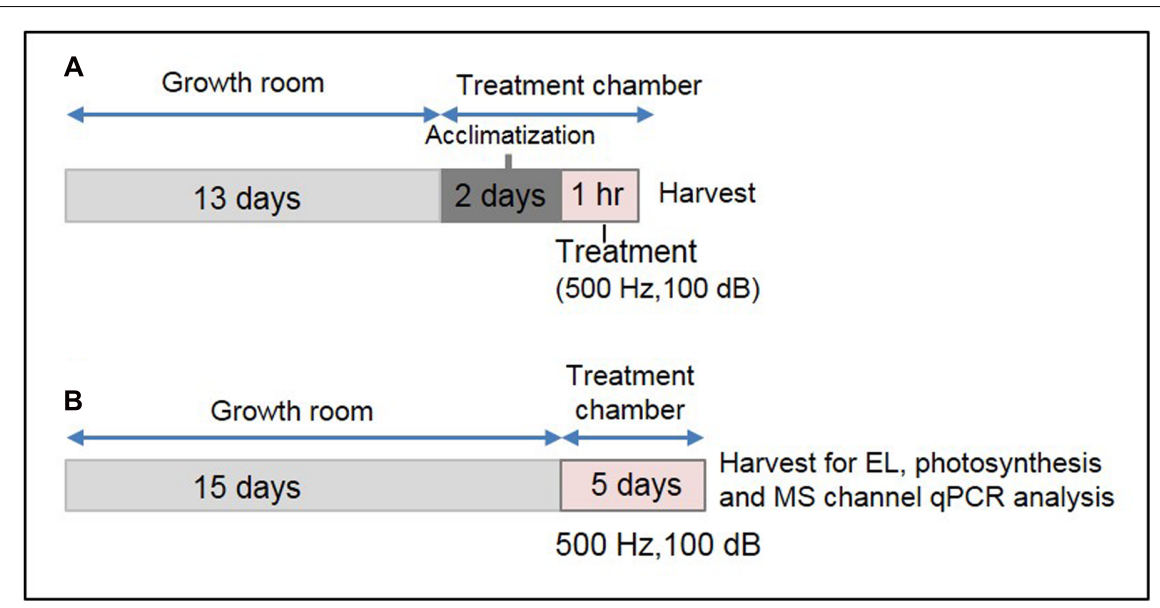

FIGURE 1 | Experimental set-up for SV treatment under lighted condition and the sample harvest strategy. (A) Schematic representation of $1 \mathrm{~h}$ SV treatment. Thirteen-day-old plants were acclimatized in the specialized plant growth chamber. Five-hundred hertz (Hz) was applied to Arabidopsis for $1 \mathrm{~h}$ with 100 decibel (dB) sound intensity. Two separate experiments were carried out with chamber swapping and whole experiment was carried under continuous light condition. (B) Schematic representation of SV treatment for continuous 5 days. Fifteen-day-old plants were exposed to $500 \mathrm{~Hz}$ at $100 \mathrm{~dB}$. After $5 \mathrm{days}$ samples were harvested for electrolyte leakage (EL), photosynthetic parameter and MS ion channel qRT-PCR analyses. 
plates were kept at $4^{\circ} \mathrm{C}$ for 2 days in dark before transferring them to the growth room. Seeds were allowed to germinate, and 5-day-old seedlings were harvested.

\section{Quantitative Real-Time PCR (qRT-PCR) Analysis}

Total RNA from an Arabidopsis rosette was extracted with the RNeasy Plant Mini kit (Qiagen, USA) and treated with DNase I (Qiagen) according to the manufacturer's instruction. cDNA was prepared with $1 \mu \mathrm{g}$ RNA, using GoScript Reverse Transcription system (Promega, USA), as per manufacturer's instructions. cDNA was diluted 10-fold before using as a template for qRT-PCR analysis. qRT-PCR was performed using Mx3000P qPCR system (Agilent, USA) and LF Taq qPCR SYBR Mix (LPS Solution, Korea). Primer details of SRGs are available in Ghosh et al. (2016). Primer details of MS ion channels are available in Supplementary Table S1. At1g13440 (GAPDH) gene encoding glyceraldehyde-3-phosphate dehydrogenase was used as an internal control. We noticed the low coefficient of variation of GAPDH across SV treatments with various $\mathrm{Hz}$ at constant amplitude (Supplementary Table S2), which indicates relatively stable expression levels. Furthermore, Duncan's multiple range test also indicated that there was no significant difference between $\mathrm{C}_{T}$ values of $G A P D H$ in different $\mathrm{Hz}$ treatment at $P$-value 0.05 . $\mathrm{C}_{T}$ values for all genes of interest $\left(\mathrm{C}_{T \cdot G O I}\right)$ were normalized to the $\mathrm{C}_{T}$ values of GAPDH $\left(\mathrm{C}_{T \cdot G A P D H}\right)$ for each replication $\left[\Delta \mathrm{C}_{T}=\left(\mathrm{C}_{T \cdot G O I}\right)-\left(\mathrm{C}_{T} \cdot G A P D H\right)\right]$ as suggested previously (Schmittgen and Livak, 2008). Relative transcript levels of each gene were calculated with respect to $G A P D H$ (\% relative expression to GAPDH) using $2^{-\triangle C T}$ value $\left[2^{-\triangle C T} \times 100\right]$ and plotted in the graph (Schmittgen and Livak, 2008). To isolate RNA from other developmental stages, a similar protocol was followed. RNA from imbibed seeds was isolated using the cetyltrimethylammonium bromide (CTAB) method as mentioned previously (Ghosh et al., 2012). Mean values and standard errors were obtained from four biological replicates.

\section{Measurement of Relative EL}

Relative EL was measured as previously described by Cao et al. (2007), with slight modifications. Three to five matured rosette leaves per plants were cut, weighed $(\sim 0.1 \mathrm{~g})$ and washed with deionized water. Cut leaves were fully submerged in $20 \mathrm{ml}$ of deionized water and shaken gently in a shaker incubator for $6 \mathrm{~h}$ (at $23^{\circ} \mathrm{C}, 140 \mathrm{rpm}$ ). Subsequently, the electrical conductivity (C1) was measured by a conductivity meter (Cond 3110, Incli.Teta Con@ 325, 2CA101, Germany). Later, the samples were boiled for $15 \mathrm{~m}$ in a water bath, following which the samples were allowed to cool to normal temperature. Conductivity was measured again for the second time (C2). Finally, relative EL was calculated using C1/C2.

\section{Measurement of Photosynthetic Parameters}

Photosynthetic parameters were measured as previously described by Gururani et al. (2015). The maximum quantum of yield of photosystem II photochemistry $(F v / F m)$ and performance index (PI) were measured using a Pocket PEA chlorophyll fluorometer (Hansatech, UK) in darkness-adapted plants. Furthermore, the chlorophyll- $a$ fluorescence transients recorded in the darkness-adapted control and SV-treated Arabidopsis plants were analyzed by the so-called JIP-test (Strasser and Tsimilli-Michael, 2001) to study their structural and functional parameters that indirectly quantify the photosynthetic behavior of the experimental plants. The data are represented in the form of a radar plot which exhibits the calculated average values of the photosynthetic parameters of the control and SV-treated Arabidopsis plants. The measurements were taken with 5-7 matured rosette leaves per plants, and the average and standard error of means were calculated from 12 individual plants.

\section{RESULTS}

\section{Expression Pattern of SRGs after SV Treatment for Various Time Periods}

Arabidopsis plants were treated with $500 \mathrm{~Hz} \mathrm{SV}$ for four different time periods: $10,30 \mathrm{~m}, 1$ and $2 \mathrm{~h}$. Plants were exposed to SV under darkness in a specialized sound-proof chamber, and control plants were kept in a similar chamber without SV. Broadly, the SRGs were down-regulated after short exposures $(10$ and $30 \mathrm{~m}$ ), and up-regulated after long exposures (1 and $2 \mathrm{~h}$ ) of SV (Figure 2). The overall expression pattern was bell-shaped: started with low expression at $10 \mathrm{~m}$, followed by highest expression at $30 \mathrm{~m}$ or $1 \mathrm{~h}$, and reduced again at $2 \mathrm{~h}$. On the basis of the up-regulation pattern, we can categorize genes into two groups: (1) genes which were upregulated either at 1 or $2 \mathrm{~h}$ (CML38, TPS8, BT5, RZPF34, LHCB2, At3g07350, At2g44500 and At2g20670), and (2) the ones which were up-regulated at both time points (CYP76C6, MPK11, MYB77, RAV1, KMD1, DREB26, HSPRO2, At1g76600 and At1g25400).

\section{Cross-Confirmation of SV-Regulated Genes' Expression under Light Condition}

Darkness-mediated up-regulation of 14 out of 17 genes was noted previously (Lee et al., 2005), which is summarized in Supplementary Table S3. Cross-talk between light/dark transition and SV treatment could be the reason for initial down-regulation (at 10 and $30 \mathrm{~m}$ ) of SRGs (Figure 2). Therefore it was necessary to confirm SRGs' expression under lighted condition. For this purpose, plants were exposed to $500 \mathrm{~Hz}$ for $1 \mathrm{~h}$ under lighted condition in a specialized plant growth chamber (Figure 1A). Similarly, to reduce the effect of movement of plants from growth room to chamber, we acclimatized the plants in the chamber for 2 days prior to SV exposure (Figure 1A). Experiments under lighted condition also confirmed the SV-mediated induction of these genes (Figure 3). Besides that, repeating the experiments with chamber-swapping conditions (marked as set 1 and 2) also confirmed the reproducibility of the set-up for future research. 


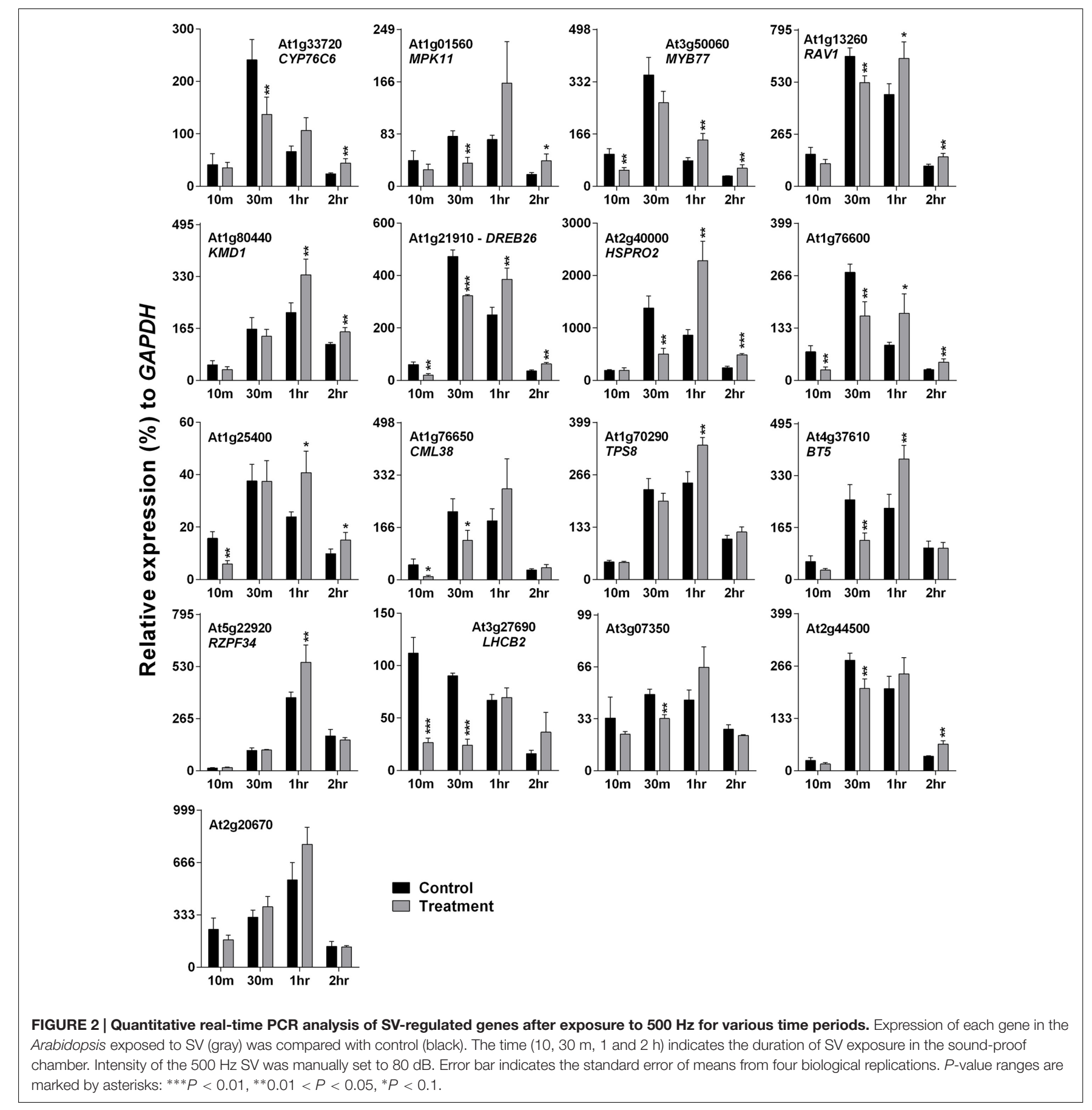

\section{Touch-Mediated Expression Profiling of SRGs}

Previously, it was hypothesized that SV and touch share some common mechanosensitive (MS) signaling events (Ghosh et al., 2016). Therefore, touch-mediated expression profiling of SRGs is necessary to elucidate their similarities and/or dissimilarities at the molecular level. Touch treatment resulted in up- and down-regulation of 11 and 4 genes, respectively, out of the 17 SRGs (Figure 4). On the basis of the expression pattern, we categorized these genes into four distinct groups: (1) genes that were highly up-regulated at $15 \mathrm{~m}$ (eight in no; At1g76600, DREB26, HSPRO2, BT5, MYB77, At1g25400, At2g44500 and $R A V 1$ ), (2) those which were down-regulated at $5 \mathrm{~m}$ and $1 \mathrm{~h}$, but up-regulated at other time points (CML38, CYP76C6 and $M P K 11),(3)$ the ones down-regulated at each time point (At3g07350, At2g20670, KMD1 and RZPF34), and (4) genes without any specific trend, but down-regulated at some time points (TPS8 and LHCB2). 


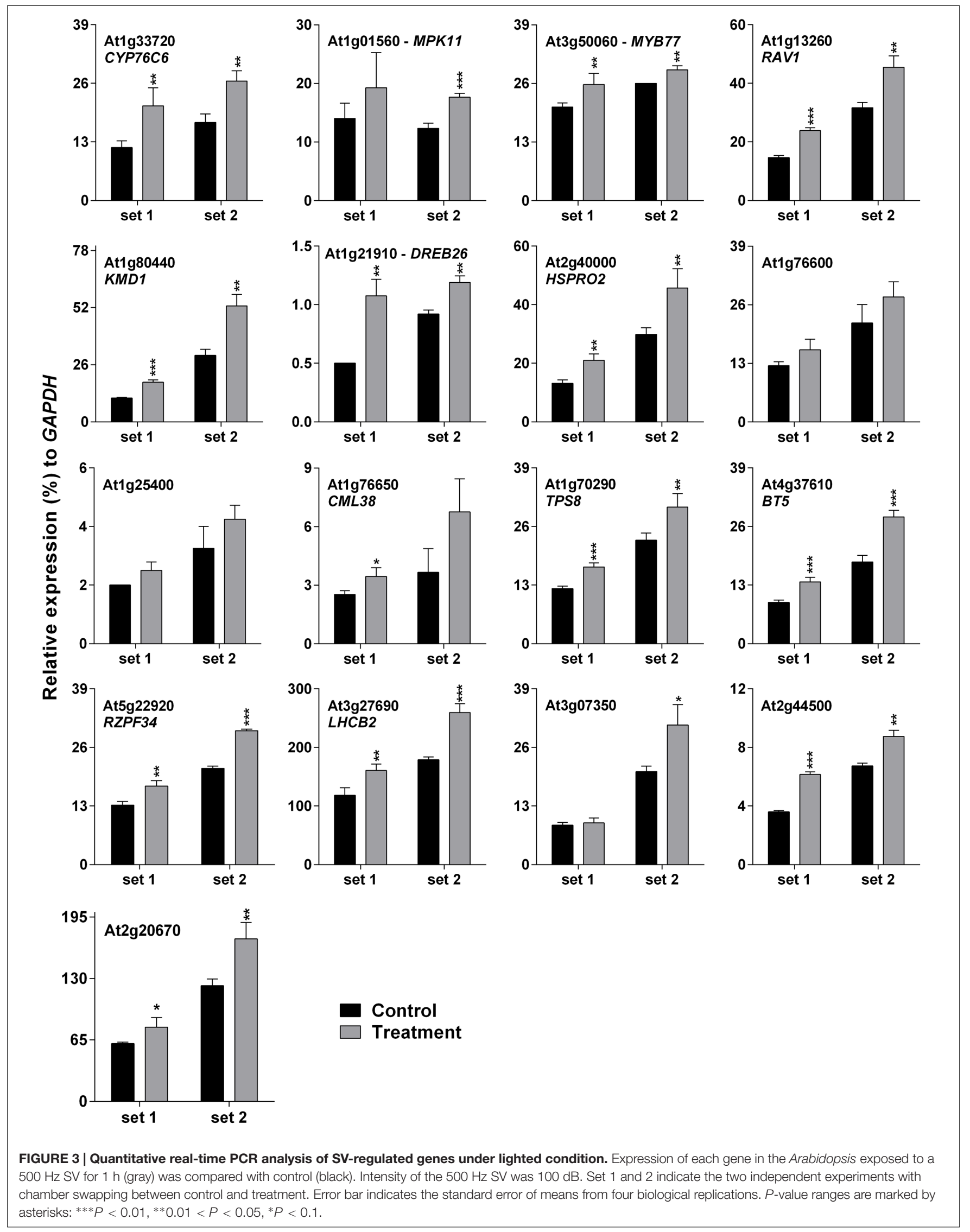




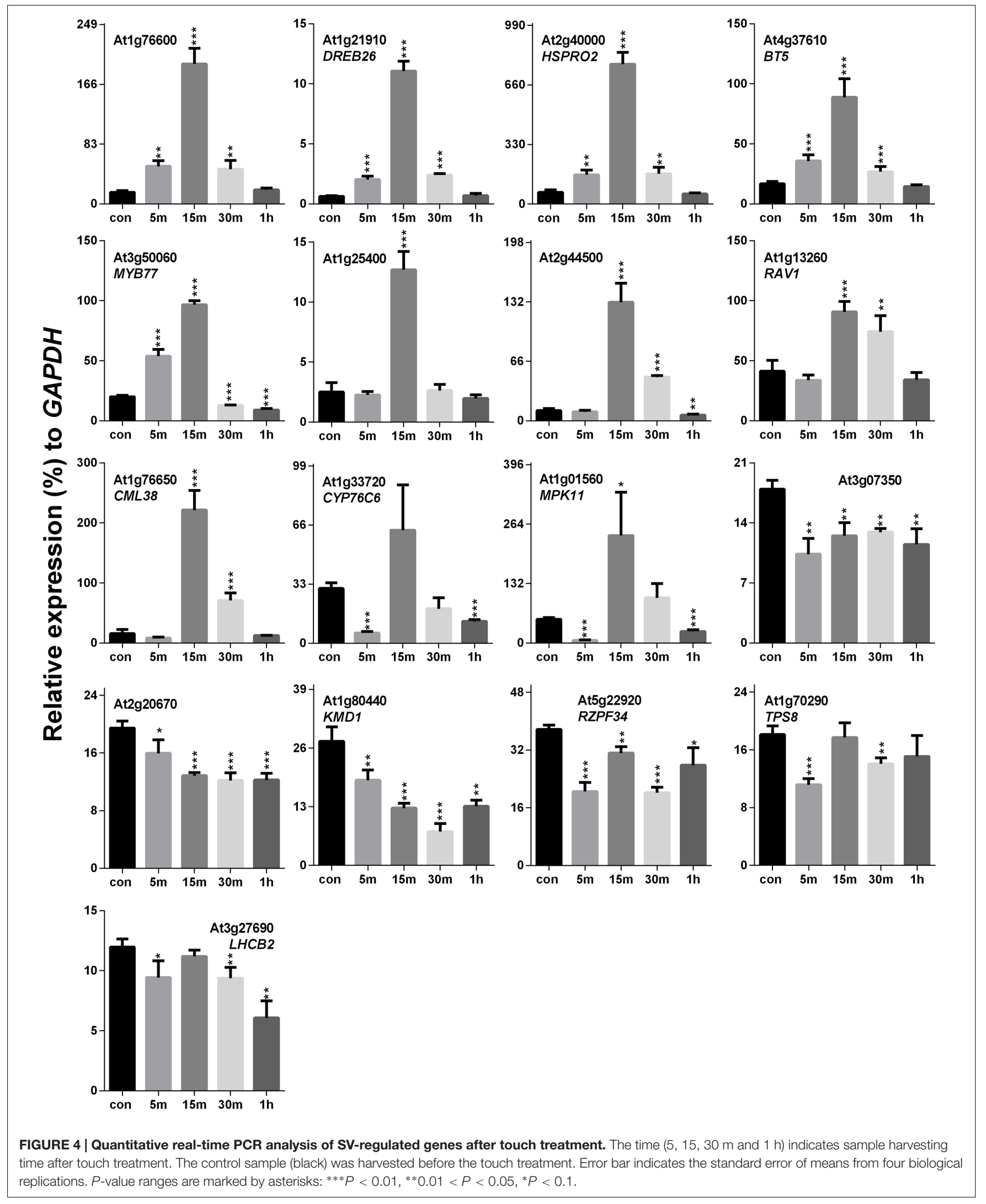




\section{Developmental Stage-Specific Expression Pattern of SRGs}

Thirteen different samples were analyzed by qRT-PCR, which are as follows: root, stem, leaves (four types; cauline, young, mature, and senescence), flowers (two types; young and mature), pods (three types; young, mature, and ripening), imbibed seeds and seedlings (Supplementary Figure S2; Supplementary Table S4). On the basis of high expression pattern, we categorized these genes broadly into four groups: (1) genes that were restricted in one developmental stage (MYB77 in imbibed seed and At3g07350 in seedlings), (2) genes that were restricted in one tissue type (LHCB2 and CYP76C6 in leaves), (3) highly expressed genes in two to three developmental stages (CML38, HSPRO2 in imbibed seeds and senescing leaves; KMD1 in seedlings and senescing leaves; At2g20670, DREB26 in seedlings and leaves; At1g25400 in young and senescing leaves; TPS 8 in imbibed seeds, seedlings and senescing leaves; At1g76600 in imbibed seeds, mature leaves and senescing leaves) and (4) highly expressed genes in more than three developmental stages (At2g44500, RZPF34, MPK11, RAV1, and $B T 5)$. However, statistically highest expression of some genes from group 2 to 4 were observed in the following developmental stages: imbibed seeds (CML38, HSPRO2 and TPS8), seedlings (KMD1, At2g20670, DREB26, RZPF34, and BT5), young leaves (CYP76C6, LHCB2, DREB26 and RAV1) and senescing leaves (At1g25400, At1g76600, and MPK11).

\section{Assessment of Changes in EL and Photosynthetic Machinery in the SV-Treated Plants}

Electrolyte leakage and photosynthetic parameters were analyzed to check the SV-mediated physiological response after 5 days of long exposure. EL is a measurement for the ionic loss through the plasma membrane caused by external stimuli (Cao et al., 2007). Higher EL was observed after 5 days of SV treatment compared to control (Figure 5A). Simultaneously, two photosynthetic parameters were measured: (i) $\mathrm{Fv} / \mathrm{Fm}$, which indicates the quantum efficiency of photosystem II and (ii) PI, which shows the PI for energy conservation from excitation to the reduction of PSI end acceptors. PI was significantly reduced after 5 days of SV treatment compared to control, though no significant change was observed in $\mathrm{Fv} / \mathrm{Fm}$ (Figure 5B). The comparative diurnal behavior of nine critical biophysical PS II characteristics in control and SV-treated plants is presented as a radar plot in Figure 5C. All data were normalized to the reference (control plants) and each variable at reference was standardized by giving it a numeric value of 1 . All variables were deduced from the JIP-test analysis. Any deviation in these parameters relative to their control values represents a change in the PSII efficiency and it has been used earlier in numerous studies (Smit et al., 2009; Gururani et al., 2015). The parameter ABS/RC indicates the total absorption of PSII antenna chlorophylls per active reactive center (RC), while $\mathrm{ET}_{0} / \mathrm{RC}$ reflects the transport of electrons to the photosynthetic electron transport chain per active PSII-RC. The trapping of an excited photon by the RCs leads to the reduction of quinone, and since at time zero, the RCs are considered open, the maximal trapping of excited photons

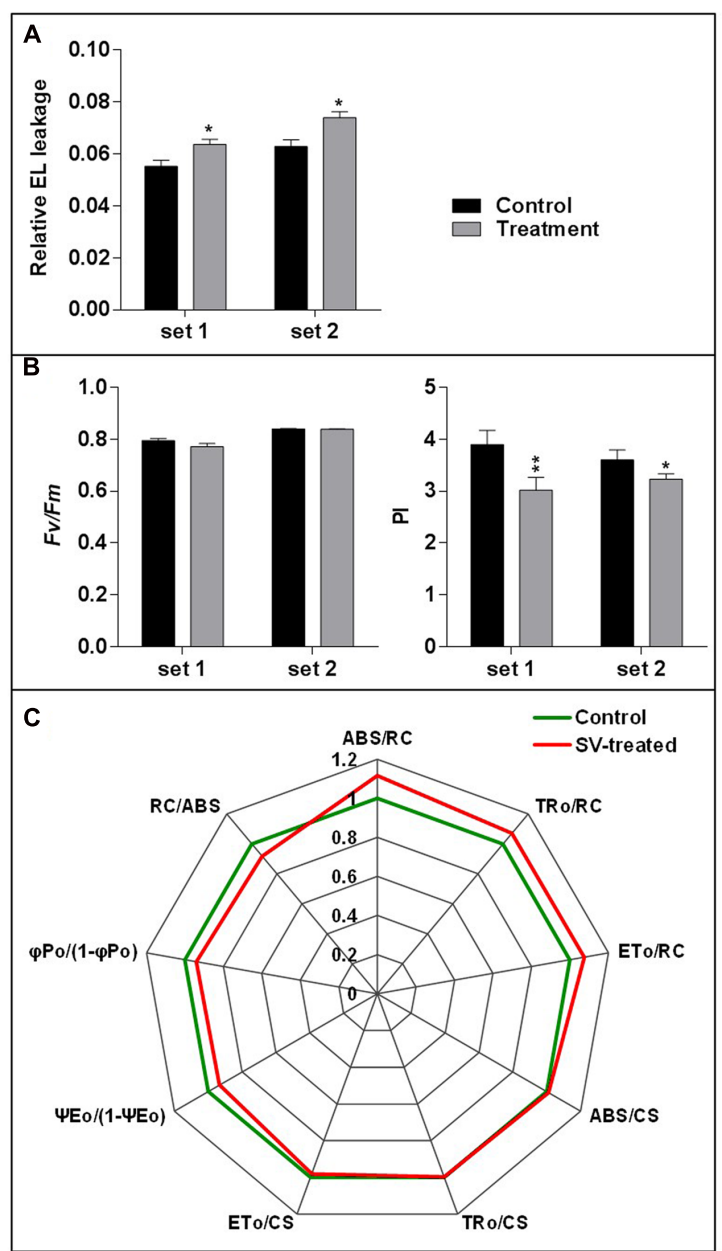

FIGURE 5 | Measurement of relative electrolyte leakage and photosynthetic parameters. (A) The relative electrolyte leakage of leaves was measured after 5 days of SV treatment $(n=10)$. (B) Similarly, maximum quantum yield of photosystem II photochemistry (Fv/Fm) and performance index $(\mathrm{Pl})$ were measured after 5 days of SV treatment $(n=12)$. Black and gray colors indicate control and SV-treated plants, respectively. Set 1 and 2 indicate the two independent experiments with chamber swapping. Error bar indicates the standard error of means. Asterisks indicate the level of significance as determined by $t$-test $\left({ }^{* *} 0.01<P<0.05,{ }^{*} P<0.1\right)$. (C) Radar plot with a series of parameters derived from JIP-test analyses of the fast OJIP transients exhibiting the differences in the structure and function of the photosynthetic apparatus in control (green) and SV-treated (red) Arabidopsis plants. The OJIP-test used in this study, delineates the maximal energy fluxes for the following photosynthetic events- absorption (ABS), trapping (TRo), and electron transport (ETo) in the energy cascade quantified by the fluxes per cross section (CS) and per reaction center $(\mathrm{RC})$ in SV-treated plants compared to those of control plants. Any marginal difference in these parameters between SV-treated and control plants is seen as a change in primary photochemistry of PSII. ABS/RC, light absorption flux (for PSIl antenna chlorophylls) per $\mathrm{RC}$; $\mathrm{TR}_{0} / \mathrm{RC}$, trapped (maximum) energy flux (leading to $\mathrm{Q}_{A}$ reduction) per $\mathrm{RC}$; $\mathrm{ET}_{0} / \mathrm{RC}$, maximum electron transport flux (further than $\mathrm{Q}_{A}-$ ) per PSII RC; ABS/CS, absorbance of photons per excited CS, TR $/ C S$, trapped energy flux per excited $\mathrm{CS}$; $\mathrm{ET}_{0} / \mathrm{CS}$, maximum electron transport flux (further than $Q_{A}-$ ) per excited CS; $\psi E$ Eo, efficiency/probability for electron transport (ET), i.e., efficiency/probability that an electron moves further than $\mathrm{Q}_{A}-$ at $t=0 ; \varphi \mathrm{Po}$, maximum quantum yield for primary photochemistry at $t=0 ; \mathrm{RC} / \mathrm{ABS}$, density of reaction centers per PSIl antenna chlorophyll. Data are mean \pm SE $(n=4, P$-value for comparison of treatments: $<0.05)$. 
per $\mathrm{RC}$ can be represented as $\mathrm{TR}_{0} / \mathrm{RC}$. The increased value of $\mathrm{ABS} / \mathrm{RC}, \mathrm{ET}_{0} / \mathrm{RC}$ and $\mathrm{TR}_{0} / \mathrm{RC}$ in $\mathrm{SV}$-treated plants suggested that the absorption and trapping flux of photons, as well as the electron transport per active RCs was higher in these plants compared to those in control plants. Notably, a concomitant increase in $\mathrm{ABS} / \mathrm{RC}$ is seen as an increase in the apparent size of the antenna rather than a structural increase in the antenna size of a biochemical complex (Strasser et al., 2004; Smit et al., 2009; Redillas et al., 2011). Next, the phenomenological energy fluxes per excited cross-section (CS) for absorption (ABS/CS), trapping (TRo/CS) and electron transport (ETo/CS) were calculated. We found that the amount of chlorophyll per leaf area of the tested samples was either similar or marginally higher than the control plants after 5 days of SV treatment. The parameter $\Psi E_{0} /\left(1-\Psi E_{0}\right)$ which indicates the efficiency of a trapped exciton to transport an electron into the photosynthetic electron transport chain was recorded to be lower in SV-treated plants than in control plants. Reduced values of $\Psi \mathrm{E}_{0} /\left(1-\Psi \mathrm{E}_{0}\right)$ with increasing salt concentration in wheat plants and in coldstressed turf grasses have been reported earlier (Mehta et al., 2010; Gururani et al., 2016). Similarly, the maximum yield of primary photochemistry $\varphi \mathrm{Po} /(1-\varphi \mathrm{Po})$ where $\varphi \mathrm{Po}=\mathrm{TRo} / \mathrm{ABS}$ was also recorded to be lower in SV-treated plants compared to that in control plants, SV treatment negatively influenced these parameters after 5 days of SV treatment. This could perhaps be corroborated with the reduced PI values in SV-treated plants (Figure 5B). PI is an indirect indicator of the vitality of plant samples where a reduced vitality is expressed in terms of reduced PI values. However, earlier studies have indicated that since electron flux is used in both carbon metabolism and other biochemical pathways, PI values are only indirectly related to the net photosynthesis (Bussotti et al., 2007). PI essentially includes three photosynthetic parameters: (1) the density of reaction centers (RC/ABS); (2) the quantum yield of primary photochemistry of PSII $(\varphi \mathrm{Po}=\mathrm{TRo} / \mathrm{RC})$, and (3) ability to feed electrons into the photosynthetic electron transport chain between PSII and PSI ( $\Psi_{0}=$ ETo/TRo) (Strasser and TsimilliMichael, 2001; Bussotti et al., 2007).

\section{Expression Analysis of MS Ion Channels after SV and Touch Treatment}

Higher EL in SV-treated plants can be caused by the modulation of membrane integrity or alteration of channel activity. Therefore, expression analysis of MS ion channels after SV treatment could be interesting. Herein we investigated the expression pattern of 13 MS ion channels after 5 days of SV treatment which are as follows: MSLs (1-10), MCAs (1 and 2) and Piezo. Among them, seven genes had a similar expression pattern between chamber swapping experiments. Four genes (MSL3, MSL4, MSL7 and MSL8) were up-regulated, and three genes (MSL10, MCA2 and PIEZO) were down-regulated in the SV-treated plant (Figure 6). Simultaneously, we checked the expression of MS ion channels after touch treatment for comparing two mechanostimuli: SV and touch. Four genes (MSL3, MSL7, MSL9 and MCA2) were up-regulated by 5 days of touch treatments in two independent experiments (Figure 7).
Among them, MSL3 and MSL7 had similar up-regulation patterns between touch treatment and SV treatment, but MCA2 showed an opposite expression pattern: down-regulated after SV treatment and up-regulated after touch treatment. The rest of the genes which showed inconsistent results between two sets of independent experiments after mechanical stimulation have been shown in Supplementary Figure S3.

\section{DISCUSSION}

The expression pattern of SRGs after exposure to SV for four different time periods in darkness was revealed (Figure 2). Upregulation was observed after long exposure (1 and $2 \mathrm{~h}$ ) of $\mathrm{SV}$, though the magnitude of expression was reduced at $2 \mathrm{~h}$. Surprisingly, the short exposure $(10$ and $30 \mathrm{~m}$ ) of SV downregulated the genes compared to the control. Previously strong correlation between darkness and touch treatments at transcript level was reported (Lee et al., 2005). More than 50\% of touchand darkness-induced genes were common (Lee et al., 2005). On the basis of our previous report as well, many up-regulated genes were common between touch and SV treatments (Ghosh et al., 2016). Among the SRGs, 14 were already noted to be up-regulated by $30 \mathrm{~m}$ of darkness, which are marked in Supplementary Table S3 (Lee et al., 2005). Besides that, nine genes (MYB77, DREB26, HSPRO2, RAV1, MPK11, At3g07350, At2g44500, At1g76600 and At1g25400) were common in touch- and SV-mediated induction (Supplementary Table S3). It is reported that both light/dark transition and touch treatments are capable of altering membrane potential in plants (Koselski et al., 2008; Chehab et al., 2011). Besides, we hypothesized that both touch and SV have a common mechanical impact in general (Ghosh et al., 2016). Previously, slight difference in transcript levels of fructose 1, 6-bisphosphate aldolase (ald) and rubisco small sub-unit $(r b c S)$ genes were noticed after the SV treatment between light- and dark- grown rice plant (Jeong et al., 2008). Collectively, our result generates a strong notion of molecular cross-talk between touch, SV and light/dark stimuli. In the future, a detailed study is needed to establish a strong correlation between these three stimuli.

The sudden transfer of light-grown plants to darkness and exposure to SV could create antagonistic molecular events. Consequently, dark and SV treatment together resulted in initial down-regulation (at 10 and $30 \mathrm{~m}$ ) of SRGs as compared to the non-competitive dark effect in the control plants (Figure 2). Nevertheless, SV exposure for a long time overcame the dark acclimatization and resulted in eventual induction of the SRGs. Simultaneously, movement of plants from growth room to the sound-proof chambers may provoke molecular alteration in plants (Figure 2). Hence, it was necessary to confirm the expression of these genes in acclimatized plants by exposure to SV under lighted condition. Swapping experiments in the illuminated chamber confirmed the SV-induced modulation of these genes (Figure 3). Additionally, the light-equipped SV treatment chamber proved to be useful for long-term SV treatment in future research. Of course, a gene transcription can be modulated by multiple environmental stimuli. Correlating a gene's expression with a particular stimulus definitely needs 

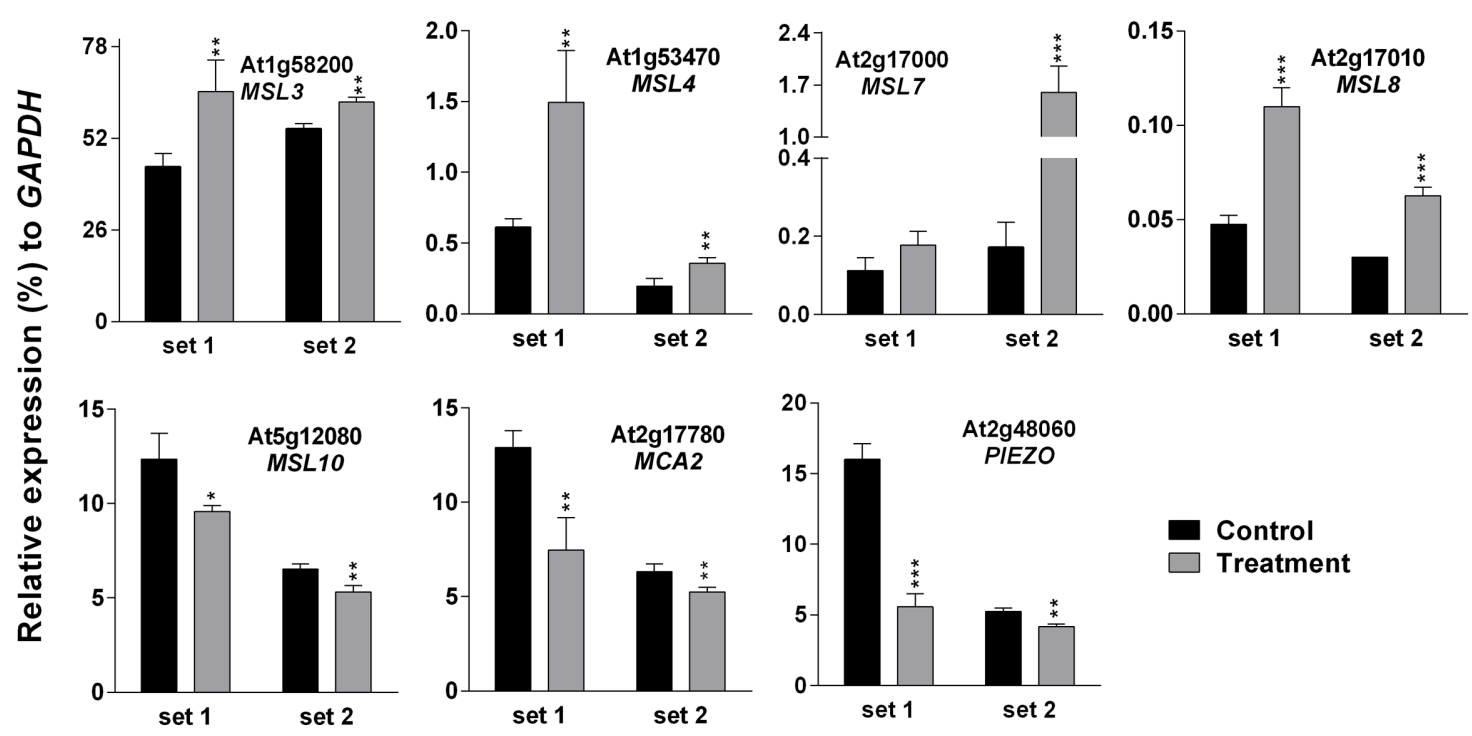

Control

Treatment

FIGURE 6 | Quantitative real-time PCR analysis of MS ion channel genes after exposure to SV. Plants were exposed to $500 \mathrm{~Hz}$ SV with $100 \mathrm{~dB}$ intensity for 5 days in a specialized plant growth chamber. Expression of each gene in the Arabidopsis exposed to SV (gray) was compared with control (black). Set 1 and 2 indicate the two independent experiments with chamber swapping between control and treatment. Error bar indicates the standard error of means from four biological replications. $P$-value ranges are marked by asterisks: ${ }^{* * *} P<0.01,{ }^{* *} 0.01<P<0.05,{ }^{*} P<0.1$.

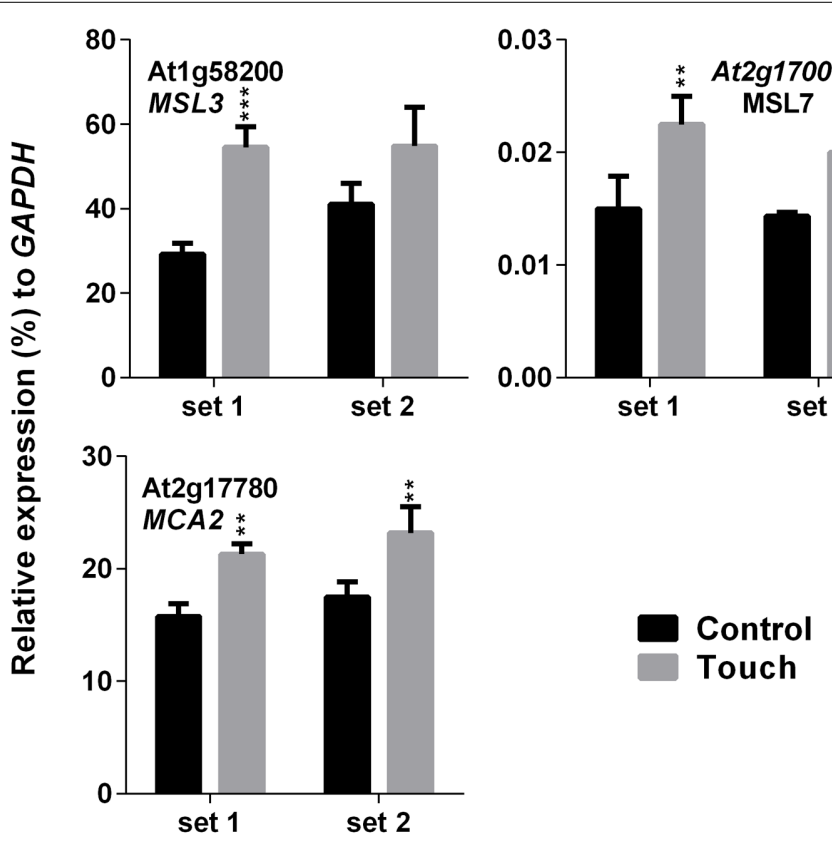

FIGURE 7 | Quantitative real-time PCR analysis of MS ion channel genes after repetitive touch treatments. Plants were repeatedly touched for 5 days. Expression of each gene in the Arabidopsis treated with touch (gray) was compared with control (black). Set 1 and 2 indicate the two independent experiments. Error bar indicates the standard error of means from four biological replications. $P$-value ranges are marked by asterisks: ${ }^{* * *} P<0.01,{ }^{* *} 0.01<P<0.05$.

rigorous study at the molecular level. Therefore, mechanistic investigation in future may shed more lights on the SV-mediated regulation of these genes.

The majority of the SRGs were expressed spatiotemporally (Supplementary Figure S2). Among them, various genes are involved in plant growth and development. SV-mediated plant growth promotion was observed previously (Hassanien et al., 2014). These genes might have a role in SV-mediated plant growth promotion. KMD1 and DREB26 encode a kelch repeat containing F-box protein and an AP2/ERF family protein, respectively. BT5 protein contains BTB and TAZ domain together. Several members of F-box, BT and AP2/ERF family 
are involved in the various processes of plant development (Kuroda et al., 2002; Robert et al., 2009; Krishnaswamy et al., 2011). CYP76C6 is a member of the cytochrome P450 subfamily C. P450s are involved in the phytohormone metabolic process and regulate plant growth and development (Xu et al., 2015). RAV1, an AP2/EREBP (APETALA2) type of TF involves in the flowering and senescence processes (Matias-Hernandez et al., 2014). Besides plant growth promotion, SV can induce the process of seed germination as well (Hassanien et al., 2014). The genes which were highly expressed in imbibed seeds probably have roles in SV-mediated seed germination. MYB77 is believed to interact with auxin response factors (ARFs) and involve in auxin response (Shin et al., 2007). Auxin is a positive regulator of gibberellic acid (GA) response and biosynthesis (Weiss and Ori, 2007); as GA is an important hormone for seed germination, this may be a reason behind SV-mediated enhancement in seed germination. $\mathrm{Ca}^{2+}$ fluxes are also considered to be crucial players during the germination process (Duval et al., 2002). $\mathrm{Ca}^{2+}$ binding calmodulin (CaM) has a role in sequestering cellular $\mathrm{Ca}^{2+}$ to maintain physiological range and avoid cytotoxicity (Duval et al., 2002). Induction of CaM transcripts was observed in pea during seed imbibition and germination (Duval et al., 2002). Therefore, it could be assumed that CaM-like (CML38) protein might be involved in similar processes. TPS enzyme converts glucose-6$\mathrm{P}$ to trehalose-6-P, a positive regulator of seed germination (Tsai and Gazzarrini, 2014). Therefore, TPS8 might also be involved in the germination process. Direct correlation of these genes with SV-mediated germination or the developmental process needs detailed study in the future.

To check the effect of long exposure on a plant's physiology, $500 \mathrm{~Hz}$ at $100 \mathrm{~dB}$ was applied for 5 days in the light-equipped SV treatment chamber and the EL and photosynthetic parameters were checked (Figure 5). Changes in molecular events such as gene expression and changes in photosynthetic efficiency in plants should not be seen as isolated events. Hence, an indirect approach called chlorophyll-a fluorescence analysis was employed to determine changes in the photosynthetic efficiency in SV-treated plants. Data from these studies indicated a significant change in the photosynthetic machinery after the plants were exposed to SV treatment for 5 days (Figures 5B,C). Previously, chlorophyll-a fluorescence studies have largely been used to assess the photosynthetic performance and overall physiology of plants under abiotic stresses (Gururani et al., 2013, 2016; Zurek et al., 2014). On the basis of the present data it can be concluded that this indirect approach of evaluating photosynthetic efficiency of plants can also be used in SV-treated plants. Measurement of EL helps to understand the status of plasma membrane integrity, which can be easily affected by external stressors (Cao et al., 2007). Previous studies have shown that SV can alter cell wall and membrane microstructure which leads to a change in tension of the cell membrane (Bochu et al., 2001; Mishra et al., 2016). SV-mediated changes in lipid fluidity and protein secondary structure of plasma membrane were observed in chrysanthemum and tobacco, respectively (Zhao et al., 2002b; Yi et al., 2003). Therefore, it could be assumed that being a pressure wave, SV exerted pressure on cell wallplasma membrane microstructure and altered its integrity which resulted in higher EL compared to the control plant. Additionally, SV-mediated changes in the activity of membrane-associated channels may alter the EL. It was previously noted that SV can alter $\mathrm{K}^{+}$channel permeability and $\mathrm{H}^{+}$-ATPase activity in chrysanthemum (Zhao et al., 2002a,c). It was hypothesized that MS ion channels might be involved in the perception of the mechanical signals (Monshausen and Haswell, 2013). The role of MS ion channels for touch sensing was observed in earlier studies (Shepherd et al., 2002; Nakagawa et al., 2007). To check whether SV has any effect on the transcript level of MS ion channels, herein we investigated their expression pattern. The result showed that SV treatment continuously for 5 days upregulated four genes (MSL3, MSL4, MSL7, and MSL8) and downregulated three genes (MSL10, MCA2 and PIEZO) (Figure 6). SV-mediated differential expression of channel genes may be involved in the higher EL, and triggers the downstream signaling processes.

Touch is considered as an external mechanical force like SV. Touch-mediated gene induction pattern was broadly spiking in nature, and was normalized to control level after $1 \mathrm{~h}$ (Figure 4). Hence, time points for expression analysis after touch treatments are crucial. Surprisingly, six SRGs (At3g07350, At2g20670, KMD1, RZPF34, TPS8 and LHCB2) were not up-regulated by touch at any of the time points. On the other hand, MCA2 showed the opposite expression pattern after 5 days of SV and touch treatments, i.e., down-regulated after SV treatment and upregulated after touch (Figures 6 and 7). This variation indicates the difference between two mechanostimuli at molecular level. Therefore, these seven genes could be the interesting candidates to highlight the differences between touch- and SV-mediated molecular responses in future. It has already been noted that the expression of MSL8 and MSL9 are mainly restricted in Arabidopsis flower and root, respectively (Haswell et al., 2008; Hamilton et al., 2015). In corroboration with this, we also observed very low expression of these two genes compared to other ubiquitously expressed MS ion channel genes (like- MSL2, MSL3, MSL5, MSL6, MSL10, MCA1 and MCA2). A detailed tissue-specific analysis of MS-ion channel genes after mechanical stimulation can lead to more interesting inferences in future.

\section{CONCLUSION}

SRGs could be regulated by darkness and touch treatments. Both being mechanostimuli, touch and SV may share some common MS signaling events. Besides, the distinct expression pattern of six SRGs and MCA2 generates an idea that SV is perceived as a stimulus distinct from touch. Certainly, a detailed comparative study is required to elucidate the similarities and dissimilarities between these two mechanostimuli. Additionally, the spatiotemporal expression of SRGs could be linked to SV-mediated growth promotion and germination by extensive research in the future. Induction of chemical defense by chewing sound of caterpillar in Arabidopsis and elevated level of polyamine by natural sound in Chinese cabbage (Qin et al., 2003; Appel and Cocroft, 2014) indicate the ecological and/or environmental significance of SV to plants. Corroboratively, 
'Buzz Pollination,' a phenomenon where pollen from anthers are released at a particular frequency produced by bee's buzz (De Luca and Vallejo-Marin, 2013), also highlights the environmental significance of SV. Such kind of natural SVs can be the interesting stimulus for extending the plant-acoustic research to molecular level. Therefore, comparative investigation with various ecologically significant SVs in natural photoperiod is required to give impetus to this least explored area of plant science.

\section{AUTHOR CONTRIBUTIONS}

RG performed most of the experiments. LP performed the experiment shown in Figure 6. RG and RM analyzed the data. MG analyzed the data shown in Figure 5C. RG, S-CP, M-JJ, and $\mathrm{HB}$ conceived the idea and designed the experiments. $\mathrm{HB}$

\section{REFERENCES}

Appel, H. M., and Cocroft, R. B. (2014). Plants respond to leaf vibrations caused by insect herbivore chewing. Oecologia 175, 1257-1266. doi: 10.1007/s00442-0142995-6

Bochu, W., Hucheng, Z., Yiyao, L., Yi, J., and Sakanishi, A. (2001). The effects of alternative stress on the cell membrane deformability of Chrysanthemum callus cells. Colloids Surf. B Biointerfaces 20, 321-325. doi: 10.1016/S0927-7765(00) 00181-8

Bochu, W., Jiping, S., Biao, L., Jie, L., and Chuanren, D. (2004). Soundwave stimulation triggers the content change of the endogenous hormone of the Chrysanthemum mature callus. Colloids Surf. B Biointerfaces 37, 107-112. doi: 10.1016/j.colsurfb.2004.03.004

Braam, J. (2005). In touch: plant responses to mechanical stimuli. New Phytol. 165, 373-389. doi: 10.1111/j.1469-8137.2004.01263.x

Bussotti, F., Strasser, R. J., and Schaub, M. (2007). Photosynthetic behavior of woody species under high ozone exposure probed with the JIP-test: a review. Environ. Pollut. 147, 430-437. doi: 10.1016/j.envpol.2006.08.036

Cao, W. H., Liu, J., He, X. J., Mu, R. L., Zhou, H. L., Chen, S. Y., et al. (2007). Modulation of ethylene responses affects plant salt-stress responses. Plant Physiol. 143, 707-719. doi: 10.1104/pp.106.094292

Chehab, E. W., Wang, Y., and Braam, J. (2011). "Mechanical force responses of plant cells and plants," in Mechanical Integration of Plant Cells and Plants, ed. P. Wojtaszek (Berlin: Springer), 173-194.

Chehab, E. W., Yao, C., Henderson, Z., Kim, S., and Braam, J. (2012). Arabidopsis touch-induced morphogenesis is jasmonate mediated and protects against pests. Curr. Biol. 22, 701-706. doi: 10.1016/j.cub.2012.02.061

De Luca, P. A., and Vallejo-Marin, M. (2013). What's the 'buzz' about? The ecology and evolutionary significance of buzz-pollination. Curr. Opin. Plant Biol. 16, 429-435. doi: 10.1016/j.pbi.2013.05.002

Duval, F. D., Renard, M., Jaquinod, M., Biou, V., Montrichard, F., and Macherel, D. (2002). Differential expression and functional analysis of three calmodulin isoforms in germinating pea (Pisum sativum L.) seeds. Plant J. 32, 481-493. doi: 10.1046/j.1365-313X.2002.01409.x

Ghosh, R., Choi, B. S., Jeong, M. J., Bae, D. W., Shin, S. C., Park, S. U., et al. (2012). Comparative transcriptional analysis of caffeoyl-coenzyme A 3-Omethyltransferase from Hibiscus cannabinus L., during developmental stages in various tissues and stress regulation. Plant Omics 5, 184-193.

Ghosh, R., Mishra, R. C., Choi, B., Kwon, Y. S., Bae, D. W., Park, S. C., et al. (2016). Exposure to sound vibrations lead to transcriptomic, proteomic and hormonal changes in Arabidopsis. Sci. Rep. 6:33370. doi: 10.1038/srep33370

Gururani, M. A., Ganesan, M., Song, I. J., Han, Y., Kim, J. I., Lee, H. Y., et al. (2016). Transgenic turfgrasses expressing hyperactive ser599ala phytochrome a mutant exhibit abiotic stress tolerance. J. Plant Growth Regul. 35, 11-21. doi: 10.1007/s00344-015-9502-0 supervised the experiments. RG, MG, RM, and $\mathrm{HB}$ wrote the manuscript. All the authors approved the final manuscript.

\section{FUNDING}

This work was carried out with the support of "Cooperative Research Program for Agriculture Science and Technology Development (PJ010497)" Rural Development Administration, Republic of Korea.

\section{SUPPLEMENTARY MATERIAL}

The Supplementary Material for this article can be found online at: http://journal.frontiersin.org/article/10.3389/fpls.2017.00100/ full\#supplementary-material

Gururani, M. A., Upadhyaya, C. P., Strasser, R. J., Yu, J. W., and Park, S. W. (2013). Evaluation of abiotic stress tolerance in transgenic potato plants with reduced expression of PSII manganese stabilizing protein. Plant Sci. 198, 7-16. doi: 10.1016/j.plantsci.2012.09.014

Gururani, M. A., Venkatesh, J., Ganesan, M., Strasser, R. J., Han, Y., Kim, J. I., et al. (2015). In vivo assessment of cold tolerance through chlorophyll-a fluorescence in transgenic zoysiagrass expressing mutant phytochrome A. PLoS ONE 10:e0127200. doi: 10.1371/journal.pone.0127200

Hamilton, E. S., Jensen, G. S., Maksaev, G., Katims, A., Sherp, A. M., and Haswell, E. S. (2015). Mechanosensitive channel MSL8 regulates osmotic forces during pollen hydration and germination. Science 350, 438-441. doi: 10.1126/science. aac6014

Hassanien, R. H. E., Hou, T. Z., Li, Y. F., and Li, B. M. (2014). Advances in effects of sound waves on plants. J. Integr. Agric. 13, 335-348. doi: 10.1016/S20953119(13)60492-X

Haswell, E. S., and Meyerowitz, E. M. (2006). MscS-like proteins control plastid size and shape in Arabidopsis thaliana. Curr. Biol. 16, 1-11. doi: 10.1016/j.cub. 2005.11.044

Haswell, E. S., Peyronnet, R., Barbier-Brygoo, H., Meyerowitz, E. M., and Frachisse, J. M. (2008). Two MscS homologs provide mechanosensitive channel activities in the Arabidopsis root. Curr. Biol. 18, 730-734. doi: 10.1016/j.cub.2008. 04.039

Jeong, M. J., Shim, C. K., Lee, J. O., Kwon, H. B., Kim, Y. H., Lee, S. K., et al. (2008). Plant gene responses to frequency-specific sound signals. Mol. Breed. 21, 217-226. doi: 10.1007/s11032-007-9122-x

Koselski, M., Trebacz, K., Dziubinska, H., and Krol, E. (2008). Light- and darkinduced action potentials in Physcomitrella patens. Plant Signal. Behav. 3, 13-18. doi: $10.4161 /$ psb.3.1.4884

Krishnaswamy, S., Verma, S., Rahman, M. H., and Kav, N. N. (2011). Functional characterization of four APETALA2-family genes (RAP2.6, RAP2.6L, DREB19 and DREB26) in Arabidopsis. Plant Mol. Biol. 75, 107-127. doi: 10.1007/s11103010-9711-7

Kuroda, H., Takahashi, N., Shimada, H., Seki, M., Shinozaki, K., and Matsui, M. (2002). Classification and expression analysis of Arabidopsis F-box-containing protein genes. Plant Cell Physiol. 43, 1073-1085. doi: 10.1093/pcp/pcf151

Lee, D., Polisensky, D. H., and Braam, J. (2005). Genome-wide identification of touch- and darkness-regulated Arabidopsis genes: a focus on calmodulin-like and XTH genes. New Phytol. 165, 429-444. doi: 10.1111/j.1469-8137.2004. 01238.x

Matias-Hernandez, L., Aguilar-Jaramillo, A. E., Marin-Gonzalez, E., SuarezLopez, P., and Pelaz, S. (2014). RAV genes: regulation of floral induction and beyond. Ann. Bot. 114, 1459-1470. doi: 10.1093/aob/mcu069

Mehta, P., Jajoo, A., Mathur, S., and Bharti, S. (2010). Chlorophyll a fluorescence study revealing effects of high salt stress on Photosystem II in wheat leaves. Plant Physiol. Biochem. 48, 16-20. doi: 10.1016/j.plaphy.2009.10.006 
Mishra, R. C., Ghosh, R., and Bae, H. (2016). Plant acoustics: in the search of a sound mechanism for sound signaling in plants. J. Exp. Bot. 67, 4483-4494. doi: 10.1093/jxb/erw235

Monshausen, G. B., and Haswell, E. S. (2013). A force of nature: molecular mechanisms of mechanoperception in plants. J. Exp. Bot. 64, 4663-4680. doi: $10.1093 /$ jxb/ert204

Nakagawa, Y., Katagiri, T., Shinozaki, K., Qi, Z., Tatsumi, H., Furuichi, T., et al. (2007). Arabidopsis plasma membrane protein crucial for $\mathrm{Ca}^{2+}$ influx and touch sensing in roots. Proc. Natl. Acad. Sci. U.S.A. 104, 3639-3644. doi: 10.1073/pnas. 0607703104

Qin, Y. C., Lee, W. C., Choi, Y. C., and Kim, T. W. (2003). Biochemical and physiological changes in plants as a result of different sonic exposures. Ultrasonics 41, 407-411. doi: 10.1016/S0041-624X(03)00103-3

Redillas, M. C. F. R., Strasser, R. J., Jeong, J. S., Kim, Y. S., and Kim, J. K. (2011). The use of JIP test to evaluate drought-tolerance of transgenic rice overexpressing OsNAC10. Plant Biotechnol. Rep. 5, 169-175. doi: 10.1007/s11816-011-0170-7

Robert, H. S., Quint, A., Brand, D., Vivian-Smith, A., and Offringa, R. (2009). BTB and TAZ domain scaffold proteins perform a crucial function in Arabidopsis development. Plant J. 58, 109-121. doi: 10.1111/j.1365-313X.2008.03764.x

Safari, M., Ghanati, F., Behmanesh, M., Hajnorouzi, A., Nahidian, B., and Mina, G. (2013). Enhancement of antioxidant enzymes activity and expression of CAT and PAL genes in hazel (Corylus avellana L.) cells in response to low-intensity ultrasound. Acta Physiol. Plant. 35, 2847-2855. doi: 10.1007/s11738-0131318-6

Schmittgen, T. D., and Livak, K. J. (2008). Analyzing real-time PCR data by the comparative C(T) method. Nat. Protoc. 3, 1101-1108. doi: 10.1038/nprot. 2008.73

Shepherd, V. A., Beilby, M. J., and Shimmen, T. (2002). Mechanosensory ion channels in charophyte cells: the response to touch and salinity stress. Eur. Biophys. J. 31, 341-355. doi: 10.1007/s00249-002-0222-6

Shin, R., Burch, A. Y., Huppert, K. A., Tiwari, S. B., Murphy, A. S., Guilfoyle, T. J., et al. (2007). The Arabidopsis transcription factor MYB77 modulates auxin signal transduction. Plant Cell 19, 2440-2453. doi: 10.1105/tpc.107. 050963

Smit, M. F., Van Heerden, P. D. R., Pienaar, J. J., Weissflog, L., Strasser, R. J., and Kruger, G. H. J. (2009). Effect of trifluoroacetate, a persistent degradation product of fluorinated hydrocarbons, on Phaseolus vulgaris and Zea mays. Plant Physiol. Biochem. 47, 623-634. doi: 10.1016/j.plaphy.2009.02.003

Strasser, R. J., and Tsimilli-Michael, M. (2001). Stress in plants, from daily rhythm to global changes, detected and quantified by the JIP-test. Chim. Nouv. 75, 3321-3326.

Strasser, R. J., Tsimilli-Michael, M., and Srivastava, A. (2004). "Analysis of the Chlorophyll a fluorescence transient," in Chlorophyll Fluorescence: A Signature of Photosynthesis, Advances in Photosynthesis and Respiration, eds G. C. Papageorgiou and Govindjee (Dordrecht: Springer), 321-362.

Telewski, F. W. (2006). A unified hypothesis of mechanoperception in plants. Am. J. Bot. 93, 1466-1476. doi: 10.3732/ajb.93.10.1466

Tsai, A. Y., and Gazzarrini, S. (2014). Trehalose-6-phosphate and SnRK1 kinases in plant development and signaling: the emerging picture. Front. Plant Sci. 5:119. doi: 10.3389/fpls.2014.00119
Veley, K. M., Maksaev, G., Frick, E. M., January, E., Kloepper, S. C., and Haswell, E. S. (2014). Arabidopsis MSL10 has a regulated cell death signaling activity that is separable from its mechanosensitive ion channel activity. Plant Cell 26, 3115-3131. doi: 10.1105/tpc.114.128082

Wei, M., Yang, C. Y., and Wei, S. H. (2012). Enhancement of the differentiation of protocorm-like bodies of Dendrobium officinale to shoots by ultrasound treatment. J. Plant Physiol. 169, 770-774. doi: 10.1016/j.jplph.2012.01.018

Weiss, D., and Ori, N. (2007). Mechanisms of cross talk between gibberellin and other hormones. Plant Physiol. 144, 1240-1246. doi: 10.1104/pp.107.100370

Xiaocheng, Y., Bochu, W., and Chuanren, D. (2003). Effects of sound stimulation on energy metabolism of Actinidia chinensis callus. Colloids Surf. B Biointerfaces 30, 67-72. doi: 10.1016/S0927-7765(03)00027-4

Xiujuan, W., Bochu, W., Yi, J., Defang, L., Chuanren, D., Xiaocheng, Y., et al. (2003). Effects of sound stimulation on protective enzyme activities and peroxidase isoenzymes of Chrysanthemum. Colloids Surf. B Biointerfaces 27, 59-63. doi: 10.1016/S0927-7765(02)00038-3

Xu, J., Wang, X. Y., and Guo, W. Z. (2015). The cytochrome P450 superfamily: key players in plant development and defense. J. Integr. Agric. 14, 1673-1686. doi: 10.1016/S2095-3119(14)60980-1

Yi, J., Wang, B. C., Wang, X. J., Chuanren, D., Toyama, Y., and Sakanishi, A. (2003). Influence of sound wave on the microstructure of plasmalemma of chrysanthemum roots. Colloids Surf. B Biointerfaces 29, 109-113. doi: 10.1016/ S0927-7765(02)00154-6

Zhao, H. C., Wang, B. C., Liu, B. A., Cai, S. X., and Xi, B. S. (2002a). The effects of sound stimulation on the permeability of $\mathrm{K}^{+}$channel of Chrysanthemum callus plasma. Colloids Surf. B Biointerfaces 26, 329-333. doi: 10.1016/S0927-7765(02) 00008-5

Zhao, H. C., Wu, J., Xi, B. S., and Wang, B. C. (2002b). Effects of sound-wave stimulation on the secondary structure of plasma membrane protein of tobacco cells. Colloids Surf. B Biointerfaces 25, 29-32. doi: 10.1016/S0927-7765(01) 00294-6

Zhao, H. C., Zhu, T., Wu, J., and Xi, B. S. (2002c). Role of protein kinase in the effect of sound stimulation on the $\mathrm{PM} \mathrm{H}^{+}$-ATPase activity of Chrysanthemum callus. Colloids Surf. B Biointerfaces 26, 335-340. doi: 10.1016/S0927-7765(02)00007-3

Zurek, G., Rybka, K., Pogrzeba, M., Krzyzak, J., and Prokopiuk, K. (2014). Chlorophyll a fluorescence in evaluation of the effect of heavy metal soil contamination on perennial grasses. PLoS ONE 9:e91475. doi: 10.1371/journal. pone.0091475

Conflict of Interest Statement: The authors declare that the research was conducted in the absence of any commercial or financial relationships that could be construed as a potential conflict of interest.

Copyright (c) 2017 Ghosh, Gururani, Ponpandian, Mishra, Park, Jeong and Bae. This is an open-access article distributed under the terms of the Creative Commons Attribution License (CC BY). The use, distribution or reproduction in other forums is permitted, provided the original author(s) or licensor are credited and that the original publication in this journal is cited, in accordance with accepted academic practice. No use, distribution or reproduction is permitted which does not comply with these terms. 